\title{
Zum Wachstum der Otolithen bei Jungheringen')
}

\author{
Von G. Hempel \\ unter Mitarbeit von H. H. Trekel \\ Biologische Anstalt Helgoland \\ (Mit 10 Abbildungen und 2 Tabellen im Text)
}

\section{Einleitung}

Zur Klärung der Frage nach der Herkunft einzelner Heringe oder Heringsschwärme wird immer wieder auf die Struktur der Otolithen als Gruppenmerkmal zurückgegriffen. Eine Zusammenstellung der älteren Literatur findet sich in ErNArssons (1951) Beitrag zur Rassenanalyse an den isländischen Heringen. Das Auftreten oder Fehlen eines hyalinen Feldes im Zentrum des Otolithen ist nach Parrish \& Sharman (1958) als Merkmal zur Unterscheidung der in der nördlichen Nordsee heimischen Heringsgruppen der Spätwinter- und der Sommer/Herbstlaicher brauchbar. Für eine Analyse der verschiedenen in der mittleren und südlichen Nordsee vorkommenden Populationen, die bezüglich ihrer Laichzeit nicht so scharf getrennt sind, läßt sich nach den Beobachtungen von Postuma \& Zijlstra (1958) die Größe des hyalinen Zentralfeldes im Otolithen verwenden. So wird den im Herbst schlüpfenden Doggerbankheringen ein größeres Zentralfeld zugeschrieben als den im Winter im Kanal schlüpfenden Downsheringen. Parrish \& Sharman weisen aber auf die Fehlerquellen durch unterschiedliche Wachstumsgeschwindigkeit der Jungheringe und durch Unterschiede im Termin, zu dem die Anlage des hyalinen Zentralfeldes abgeschlossen ist, hin. Auch ist wenig über das Otolithenwachstum in Beziehung zur Körpergröße bei Jungheringen und Heringslarven bekannt. Bevor wir mit einiger Sicherheit Schlüsse aus der Größe des hyalinen Zentralfeldes auf die frühe Lebensgeschichte eines Herings und damit auf seine Abstammung von einer der Laichgemeinschaften der Nordsee ziehen können, ist eine Untersuchung über das Wachstum des Otolithen und die Entwicklung seiner Struktur während der Jungstadien erforderlich. Einige Angaben hierzu finden sich in den Arbeiten von Jenkins (1902), der an Jungheringen der Kieler Bucht, des Windebyer Noors und der Nordsee (9 Exemplare) Otolithenmessungen durchführte, und von Lissner (1925), der die Entwicklung des Heringsotolithen in großen Zügen beschreibt. Die 1957 in Angriff genommenen Arbeiten, deren erste Ergebnisse hier mitgeteilt werden, befassen sich vor allem mit den Oto-

1) Ein Teil der zu diesen Untersuchungen verwendeten Ausrüstung wurde aus Mitteln de1 Deutschen Wissenschaftlichen Kommission für Meeresforschung angeschafft. 






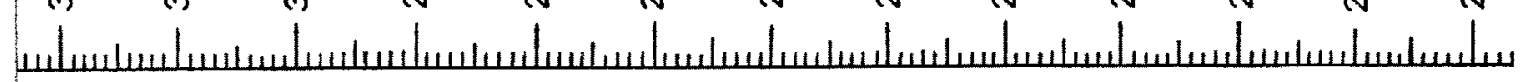
गागए

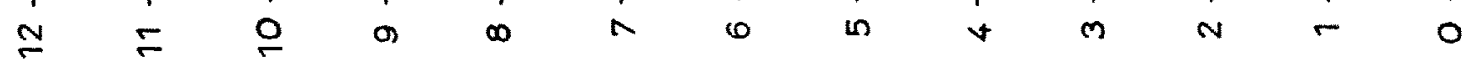

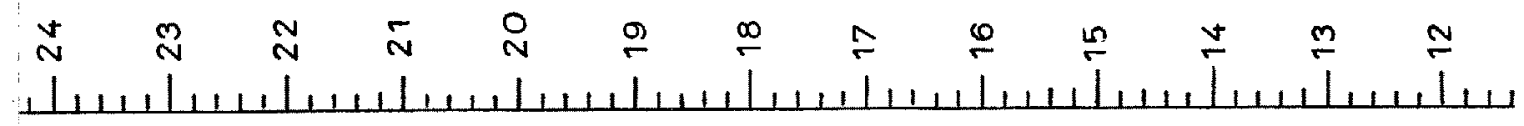

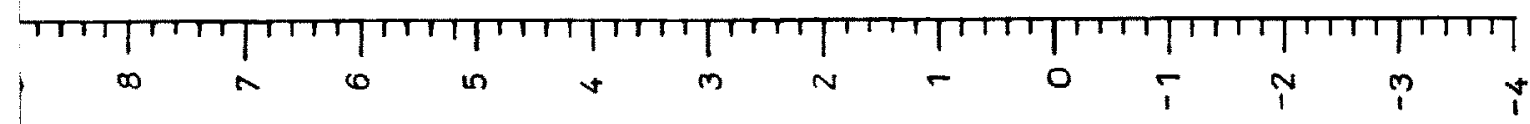
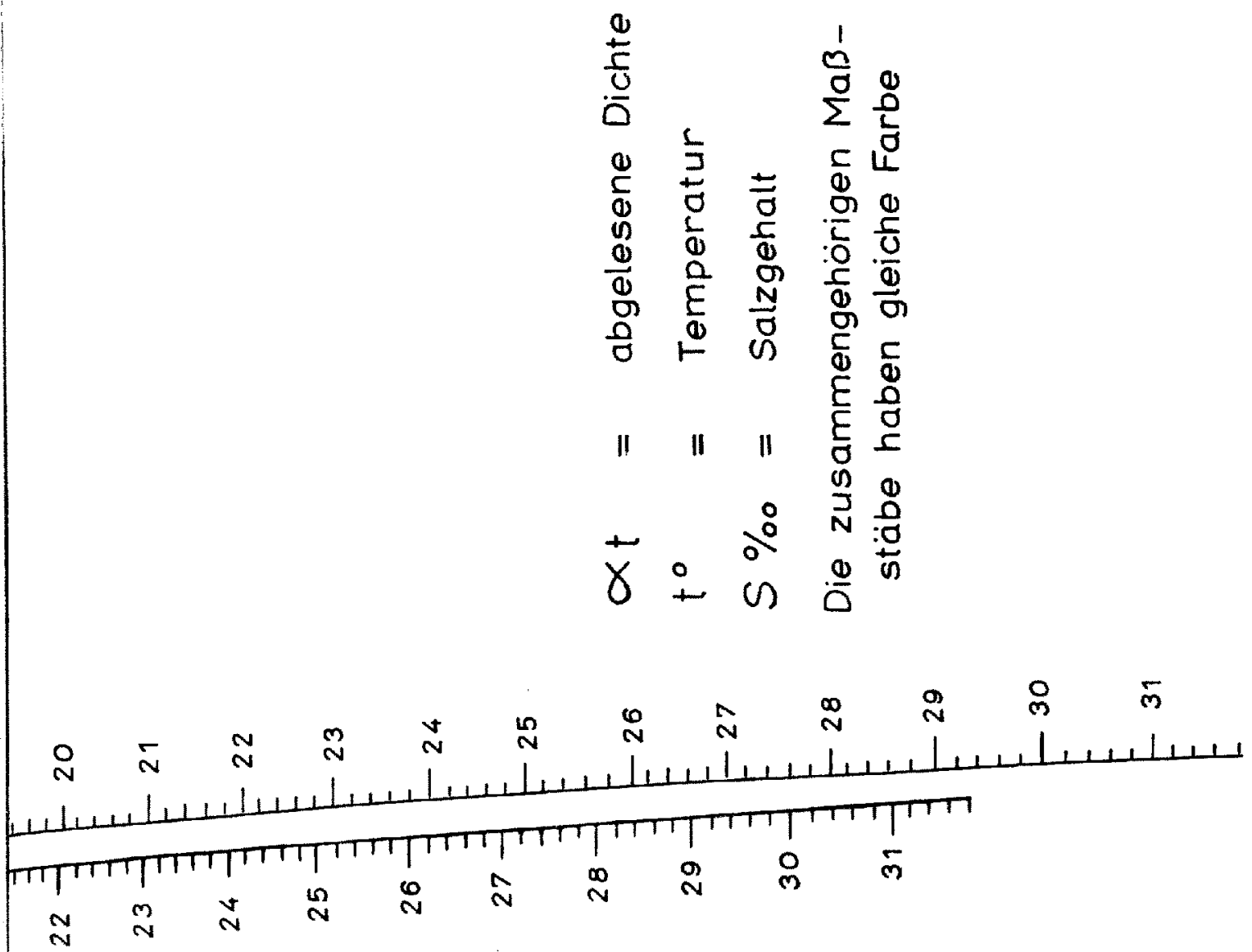
lithen jugendlicher Doggerbankheringe. In einem späteren Stadium der Untersuchungen sollen die Abkömmlinge anderer Laichgemeinschaften zum Vergleich herangezogen werden.

\section{Material und Methodik}

Die Heringslarven und Jungheringe, die dieser Untersuchung zugrunde liegen, wurden in der inneren Deutschen Bucht fast ausschließlich in den Jahren 1957 und 1958 gefangen. Eine Zusammenstellung der Fangdaten der einzelnen Proben findet sich in Tab. 1 und Abb. 1.

Die Heringe der Proben I, II, III, V, VII, IX, XIV, XV und XX stammen aus Fängen des Forschungskutters "Uthörn" der Biologischen Anstalt Helgoland. Die Proben VI, XII, XIII, XVI sammelten wir aus Trawlfängen von FFS "Anton Dohrn“, die Proben IV, VIII. XI, XVII, IXX aus in List angelandeten Fängen der Krabbenkutter, die Proben XXI und XXII verdanke ich den Bemühungen von Dr. Lühmann und Dr. KüHL. Institut für Küstenund Binnenfischerei, Hamburg und Cuxhaven, die Probe XVIII Herrn K. Lübers, „Senckenberg am Meer", Wilhelmshaven. Insgesamt wurden 1271 Heringe bearbeitet, an 903 von ibnen wurde die Otolithenlänge, an 1017 die Kopflänge bestimmt.

Tabelle 1

Liste der bearbeiteten Proben 1956-1958

\begin{tabular}{|c|c|c|c|c|c|c|}
\hline $\begin{array}{l}\text { Proben- } \\
\text { Nummer }\end{array}$ & Datum & Fangort & Fanggerät & $\begin{array}{l}\text { Zahl der } \\
\text { bearbeit. } \\
\text { Tiere }\end{array}$ & $\begin{array}{l}\text { Mittl. } \\
\text { Länge } \\
\text { mm }\end{array}$ & $\begin{array}{l}\text { Art der } \\
\text { Bearbeit. }\end{array}$ \\
\hline I & 11. 2. 57 & westl. Sylt & Knüppelnetz & 2 & 39 & $O t$ \\
\hline II & Febr./März 57 & Helgoland Rinne & Knüppelnetz & 9 & 33,2 & $\mathrm{Ot}$ \\
\hline III & 16. 2. 57 & Elbe I Fsch. & Knüppelnetz & 19 & 35,4 & $\mathrm{Ot}(6), \mathrm{K}(13)$ \\
\hline IV & Mai/Juni 57 & Sylt-Röm & Granatkurre & 32 & 64,2 & $\mathrm{~K}$ \\
\hline $\mathrm{V}$ & 3. 4.57 & Vortrapp-Tief & Knüppelnetz & 65 & 31,5 & $O t$ \\
\hline VI & 28.6 .58 & Austerngrund & Kuttertrawl & 102 & 154,4 & $\mathbf{K}$ \\
\hline VII & 22.4 .58 & Elbe III Fsch. & Knüppelnetz & 113 & 43,1 & $\mathrm{Ot}(83), \mathrm{K}(30)$ \\
\hline VIII & 9.5 .57 & Röm & Granatkurre & 80 & 59,2 & $\mathrm{Ot} \quad \mathrm{K}(73)$ \\
\hline IX & 21. 7.57 & Lister Tief & Helgol.Trawl & 1100 & 84.5 & Ot \\
\hline $\mathrm{X}$ & 15.6 .57 & List/Strand & angespült & 38 & 65,1 & $\mathrm{Ot}$ \\
\hline XI & 1. 6.57 & Sylt-Röm & Granatkurre & 19 & 70,8 & $O t$ \\
\hline XII & 27.6 .57 & Helgoland & Kuttertrawl & 55 & 97,3 & $\mathrm{Ot}(51), \mathrm{K}$ \\
\hline XIII & 5. 7.57 & sö Helgoland & Kuttertrawl & 65 & 79,9 & $\mathrm{~K}(63)$ \\
\hline XIV & 6.5 .58 & Elbmündung & Knüppelnetz & 111 & 42,0 & $\mathrm{Ot}$ \\
\hline $\mathrm{XV}$ & 19. 3. 57 & Elbe I Fsch. & Knüppelnetz & 33 & 26,1 & K \\
\hline XVI & 29. 6.58 & Clay Deep & Kuttertrawl & 127 & 161,5 & $\mathrm{Ot}(53), \mathrm{K}(120)$ \\
\hline XVII & 29.5 .58 & Röm & Granatkurre & 44 & 55,9 & $\mathrm{Ot}$ \\
\hline XVIII & Nov. 56 & Jadebusen & Senknetz & 83 & 117,6 & $O t$ \\
\hline XIX & 10.-12. 6. 58 & Sylt & Granatkurre & 29 & 69,3 & $\mathrm{Ot}$ \\
\hline $\mathrm{XX}$ & 16.5 .57 & Elbe III Fsch. & Knüppelnetz & 95 & 47,1 & $\mathrm{~K}$ \\
\hline $\mathrm{XXI}$ & $31.7 .-1.8 .58$ & Jadebusen & Granatkurre & 38 & 114.1 & Ot $(36), \mathrm{K}(38)$ \\
\hline XXII & 14.8 .58 & Elbmündung & Granatkurre & 82 & 107,8 & $\mathrm{Ot}(46), \mathrm{K}(82)$ \\
\hline
\end{tabular}

$\mathrm{O} t=$ Otolithenlänge, $\mathrm{K}=$ Kopflänge

Um zu prüfen, ob nahe der Elbmündung oder im ostfriesischen Küstenbereich gefangene Jungheringe etwa den Frühjahrslaichern in Elbe und Ems zugehören, wurden bei kleineren Proben die Wirbelzahlen bestimmt: XIII $56,54 \pm 0,14$, XVIII $56,56 \pm 0,05$ (Bückmann und Hemper 1957), XXI $56.57 \pm 0,14$, XXII $56,28 \pm 0,12$. Demnach bleibt nur bei der in der Elbmündung gewonnenen Probe XXII der Verdacht auf eine Beimischung von Elbheringen bestehen. 
Nach früheren Untersuchungen über die mittlere Körperlänge der Heringslarven verschiedener Herkunft während der Frühjahrsmonate lassen sich aus der Längenverteilung der Larven gewisse Rückschlüsse auf ihre Herkunft ziehen. In Abb. 2 ist die Größenzusammensetzung der größeren Larvenproben dargestellt. Nach Bückmann \& Hempes (1957, Abb. 1 b) waren die Doggerbanklarven in der Jade Anfang April 1949 und 1951 im Mittel ca.

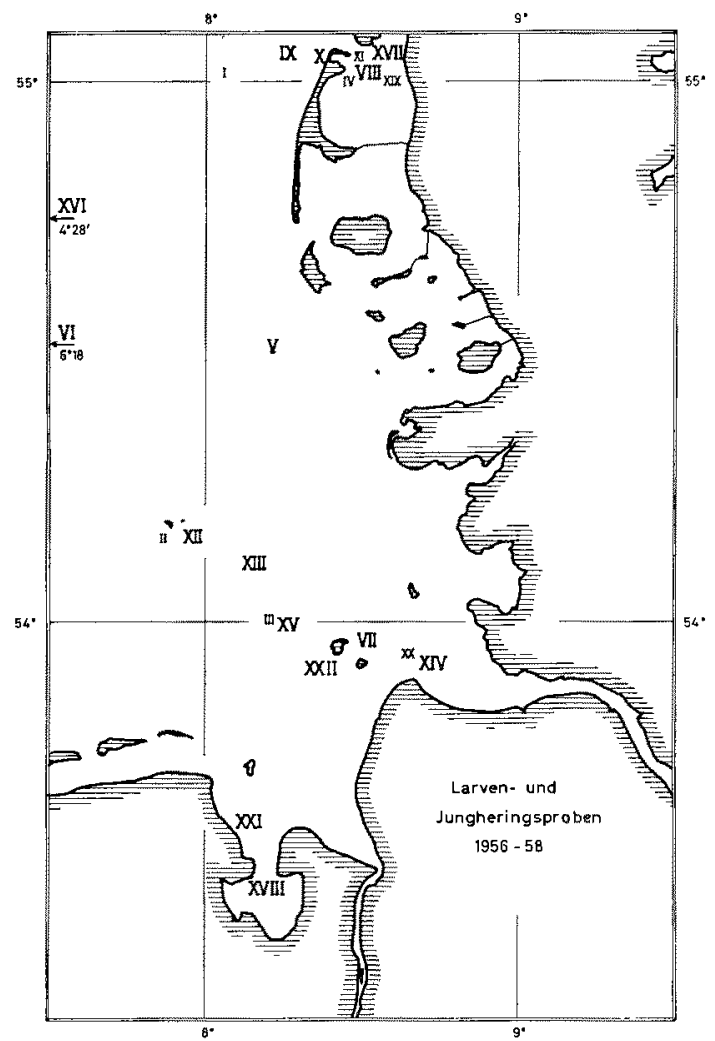

Abb. 1. Karte des Untersuchungsgebietes. Die wichtigen Proben sind durch große Ziffern hervorgehoben

33-35 mm lang, 1952 dagegen um $40 \mathrm{~mm}$. Da zuerst die schnellwüchsigen Larven in die Astuare eindringen, können wir in dem der Küste vorgelagerten Gebiet mit etwas niedrigeren Werten rechnen. Die Probe V vom 3. 4.1957 weist ihren Hauptgipfel bei $33 \mathrm{~mm}$ auf, diese Tiere stammen vermutlich von Herbstlaichern $a b$. Es muß angenommen werden, daß die Larven unter $30 \mathrm{~mm}$, die dieser Probe beigemischt waren und die z. T, auch bearbeitet wurden, Downslarven sind. Bei der Probe VII vom 22. 4. 1958 liegt das Maximum bei 43-45 mm, was nach den Erfahrungen früherer Jahre auf ein Vorherrschen von Doggerbanklarven neben kleinen Downslarven schließen läßt. Die Probe von Mitte Mai 1957 bestand wahrscheinlich aus Doggerbanktieren. $\mathrm{Daß}$ das Maximum der Längenverteilung gegenüber dem Fang von Ende April 1958 kaum verschoben ist, liegt vermutlich daran, daß nur die langsamwüchsigen Vertreter der Doggerbankgruppe Mitte Mai noch nicht metamorphisiert waren und vom Knüppelnetz erfaßt werden konnten. Die Verteilung am 6. Mai 1958 (Probe XIV) war dagegen zweigipfelig. Neben Dogger- 


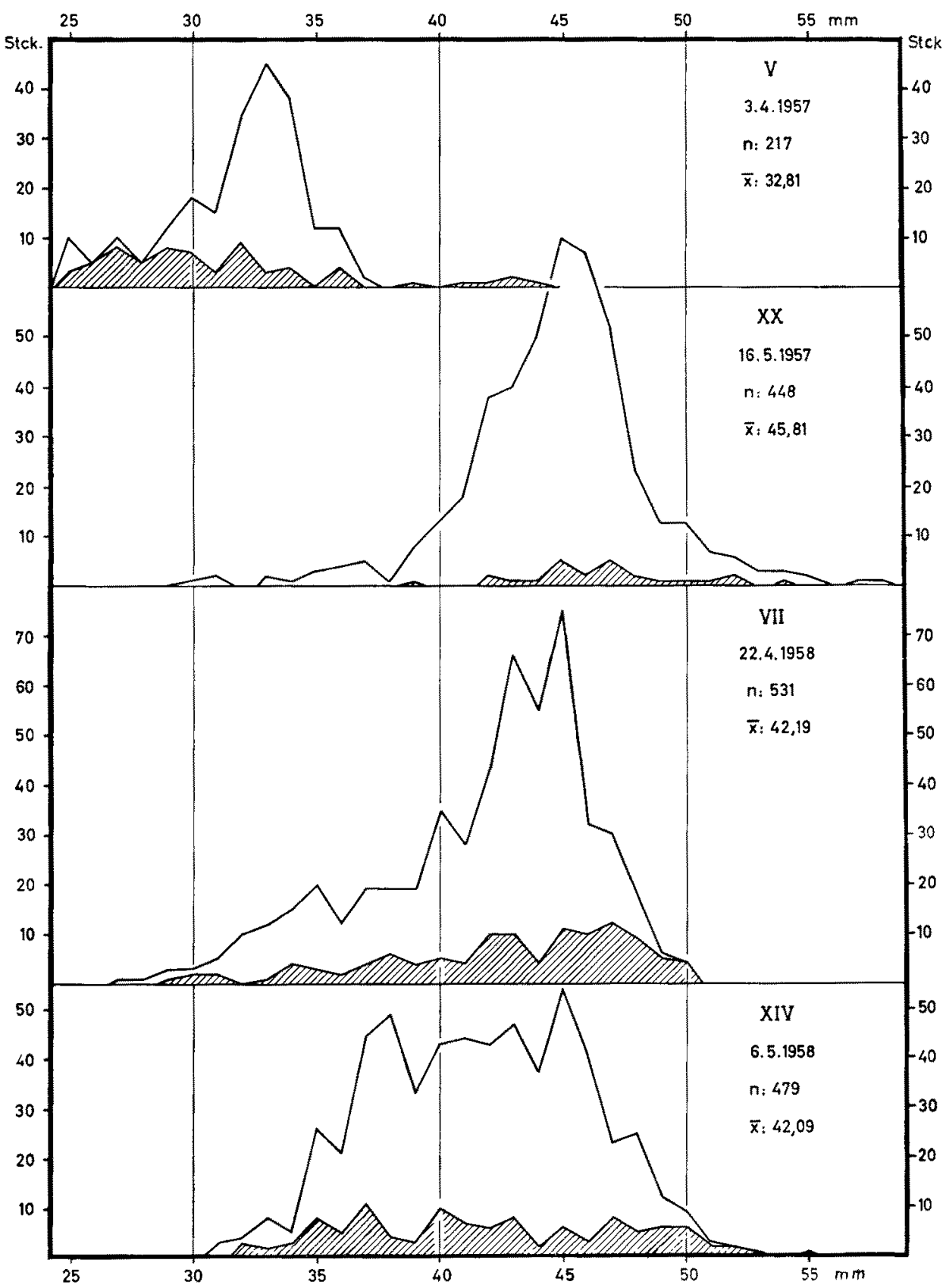

Abb. 2. Längenzusammensetzung der wichtigeren Larvenproben. Weiß: Gesamtprobe, schraffiert: bearbeitete Teilprobe

banklarven wurden viele Downslarven (etwa $41 \mathrm{~mm}$ ) angetroffen. Auf Grund ihrer Körperlängen weisen sich die bearbeiteten Larven der kleinen Proben I, II und III als Ảbkömmlinge von Sommer- und Herbstlaichern aus. Wollen wir mit einiger Sicherheit nur Doggerbanklarven bei den Betrachtungen über die Größe des Zentralfeldes behandeln, so müssen wir in den Mischproben je- 
weils die kleinsten der bearbeiteten Larven ausschalten, und zwar von Probe V 36 Larven, von Probe VII 19 Larven, von Probe XIV 56 Larven. Diese Larven erhielten bei der Darstellung der Zentralfeldgröße eine eigene Signatur. In Anbetracht der geringen morphometrischen Unterschiede zwischen den Herbst- und Winterlaichern der südlichen Nordsee wurden beim Vergleich des Wachstums von Totallänge, Kopf und Otolith die Downslarven nicht von den Doggerbanklarven gesondert. Die Jungheringe der Proben IV, VIII, XI, XIII, XVII und IXX, die im Mai bis Anfang Juli gefangen wurden, sind, soweit sie im ersten Lebensjahr stehen, größer als man für Downsheringe erwarten kann. Auch die Fangplätze vor der nordfriesischen Küste sprechen für eine Herkunft dieser Tiere von der Doggerbank und den nördlicher gelegenen Laichplätzen. Eine Entscheidung über die Herkunft der im 2. und 3. Lebensjahr stehenden großen Jungheringe, die vor allem in den Proben IX, XII, XVI, XXI und XXII enthalten sind, kann nicht gefällt werden, es darf aber vermutet werden, daß auch sie zum überwiegenden Teil von Herbstlaichern abstammen.

Die Tiere wurden in $70 \%$ Alkohol, später in $80 \%$ Alkohol fixiert und aufbewahrt. Sofern es sich um Fänge der Krabbenkutter handelte, konnten die Heringe erst einige Stunden nach dem Tode konserviert werden. Auch bei einzelnen Proben großer Heringslarven war die Fixierung anfangs unzureichend. Hier mögen geringfügige Änderungen der Körperlänge eingetreten scin. Infolge der Alkoholkonservierung schrumptten die Heringe, so daß die angegebenen Totallängen etwas zu niedrige Werte darstellen. Eine Wiederholungsmessung zeigte, daß einige Zeit nach der Fixierung die Schrumpfung zum Stillstand kommt: Es fand sich kein Unterschied in den Meßergebnissen, die ein Monat und drei Monate nach der Fixierung gewonnen worden waren. So konnten im Frühsommer 1958 unbedenklich Proben von 1957 neben solchen von 1958 bearbeitet werden.

Neben der Totallänge (zum nächstniedrigeren Millimeter) wurde bei den Heringen die Kopflänge (Schnauzenspitze bis Hinterrand des Operculum) bestimmt, bei den kleineren Tieren unter einer binokularen Lupe mit Meßokular, bei den größeren mit Hilfe eines Stechzirkels. War das Operculum bei den Larven noch nicht voll entwickelt, so wurde bis zum caudalen Rand der Kiemen gemessen. Zur Ausmessung der Otolithen wurden diese auf Kleinbildfilm photographiert. Nach dem Forttrocknen des Haftwassers, das mit dem Otolithen nach der Präparation auf den Objektträger gelangt war, wurde zu dem Otolithen Xylol gegeben. Nach 1-24 Stunden, je nach der Dicke des Otolithen, war dieser gut aufgehellt, so daß er klare Bilder lieferte. Die Otolithen von Heringen bis $70 \mathrm{~mm}$ konnten im Durchlicht, die größeren nur im Auflicht (senkrechtes Licht durch Leitz-Ultropak mit zusätzlicher Schrägbeleuchtung) photographiert werden. Die Ausmessung erfolgte unter dem Vergrößerungsapparat am Negativ. Auf jeden Film wurde einmal ein Objektmikrometer aufgenommen, dessen Bild im Vergrößerungsapparat den Millimetermaßstab für den Otolithen lieferte, so daß sich die sonst übliche Umrechnung von Skalenteilen in Millimeter erübrigte. Als Länge des Otolithen bzw. des Zentralfeldes wurde der größte Durchmesser bestimmt, der das Zentrum schneidet. Meist verläuft die Meßlinie zur Bestimmung der Otolithenlänge von der Spitze des Rostrum (oder etwas ventral davon) zum caudal/dorsalen Rand des Yararostrum. Größere Schwierigkeiten machte vielfach die Ausmessung des Zentralfeldes. Hierauf muß später noch im einzelnen eingegangen werden.

Zur statistischen Behandlung wurde das Material nach der Totallänge geordnet in 15-mm-Gruppen zusammengefaßt. Obwohl Jungheringe bis $209 \mathrm{~mm}$ Totallänge bearbeitet wurden, blieben bei den graphischen Darstel- 


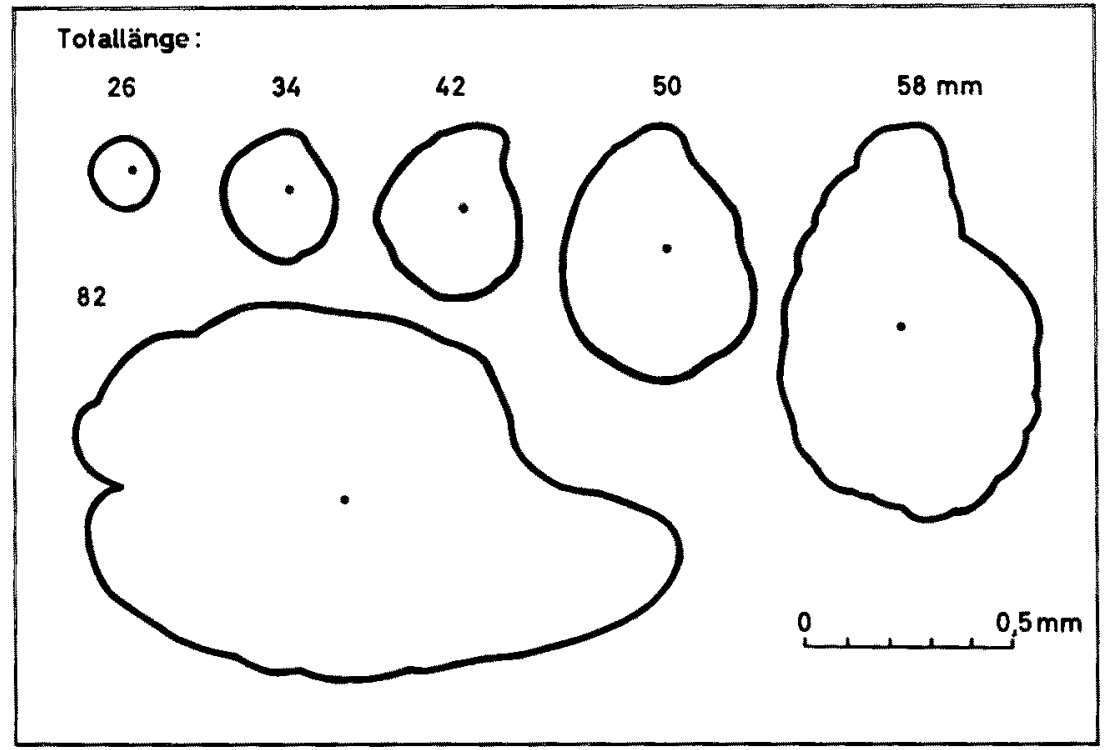

Abb. 3. Veränderungen im Umriß des Heringsotolithen während des ersten Lebensjahres. Zeichnung nach Photographien

lungen und bei den Berechnungen Tiere über $130 \mathrm{~mm}$ Totallänge unberücksichtigt. Das Beobachtungsmaterial an größeren Heringen reicht bisher nicht aus, verläßliche Angaben über das Otolithenwachstum zu machen.

\section{Das Otolithenwachstum}

\section{a. Form des Otolithen}

Die kleinsten Heringslarven, die uns zur Präparation zur Verfügung standen, waren $25 \mathrm{~mm}$ lang. Ihre Otolithen sind fast kreisrunde, uhrglasförmige Gebilde. Abb. 3 zeigt in Umrißbildern die Veränderungen der Otolithenform im Verlauf der Jugendentwicklung. Bei Tieren von etwa $90 \mathrm{~mm}$ Totallänge sind alle Charakteristika des Heringsotolithen ausgebildet. Im Verlauf des weiteren Wachstums kommt es aber noch zu einer Streckung des Otolithen, insbesondere des Rostrums. Auch erscheint bei älteren Tieren der Sulcus und die Excisura minor deutlicher und der Otolith wird stärker skulpturiert. Ausformung und Längenwachstum des Otolithen stehen nicht in enger Abhängigkeit voneinander. Gleichgroße Otolithen verschiedener Tiere weisen mitunter erhebliche Unterschiede in der Form auf. Wenig unterscheiden sich dagegen in Größe, Form und Struktur die beiden Otolithen eines Tieres. Abgesehen von Kontrollen, die bei $10 \%$ des Gesamtmaterials durchgeführt wurden, erübrigte es sich daher, jeweils beide Otolithen zu bearbeiten. Meist wurde der linke Otolith freipräpariert.

\section{b. Die Länge des Otolithen}

Die Länge des Otolithen wurde zur Totallänge des Tieres in Beziehung gesetzt und ferner in Fortsetzung der Untersuchungen von Heincke (1897) 


\section{Tabelle 2}

Allometrie von Otolith und Kopf bei jungen Heringen. a u. b Werte aus den linearen Regressionsgleichungen für die einzelnen Längengruppen. $y=a+b \cdot x$

Totallänge : Otolithenlänge

\begin{tabular}{|c|c|c|c|c|c|c|c|c|}
\hline $\begin{array}{l}\text { Totallänge } \\
\text { mm }\end{array}$ & $\mathrm{n}$ & $\underset{\mathrm{mm}}{\mathrm{a}}$ & $\mathrm{b}$ & $\mathrm{P}$ & $n$ & $\underset{\mathrm{mm}}{\mathrm{a}}$ & b & $P$ \\
\hline $25-39$ & 142 & $-0,27$ & 1,697 & $<0,001$ & 102 & $-0,23$ & 2,307 & $<0,001$ \\
\hline $40-54$ & 175 & $-0,62$ & 2,669 & & 166 & $-0,77$ & 3,732 & $<0,001$ \\
\hline $55-69$ & 128 & $-0,37$ & 2,203 & $<0,001$ & 148 & $+0,02$ & 2,261 & $\mathrm{~b}=2,44$ \\
\hline $70-84$ & 143 & $-0,99$ & 3,206 & $<0,001$ & 151 & $+0,03$ & 2,294 & $<0,001$ \\
\hline $85-99$ & 102 & $+0,95$ & 0,846 & $<0,001$ & 135 & $+0,84$ & 1,362 & $<0,001$ \\
\hline $\begin{array}{l}100-114 \\
115-130\end{array}$ & $\begin{array}{l}41 \\
35\end{array}$ & $\begin{array}{r}-2,34 \\
*)\end{array}$ & 4,117 & $b=1,78$ & $\begin{array}{l}41 \\
35\end{array}$ & $\begin{array}{l}-1,25 \\
+1,21\end{array}$ & $\begin{array}{l}3,436 \\
1,264\end{array}$ & $b=2,77$ \\
\hline
\end{tabular}

*) Keine signifikante Korrelation.

Kopflänge : Otolithenlänge

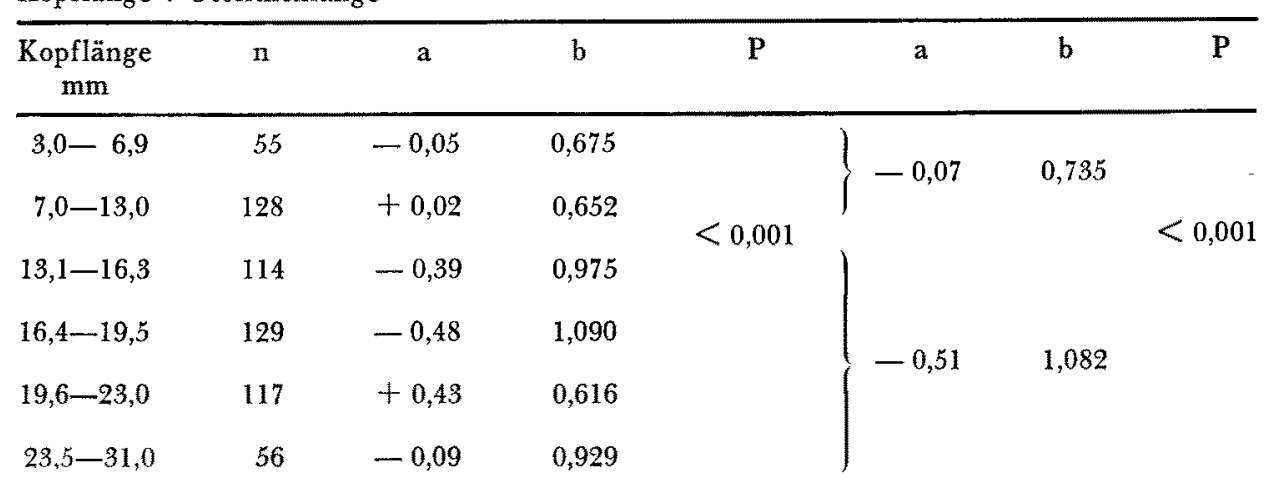

das Wachstum des Kopfes in Abhängigkeit von der Körpergröße bestimmt, da angenommen werden konnte, daß das Otolithenwachstum enger mit dem Kopfals mit dem Körperwachstum korreliert ist. Zwischen den Größenzunahmen von Totallänge, Kopf und Otolith besteht in dem hier betrachteten Größenbereich keine lineare Beziehung. Im Rahmen der geplanten Untersuchungen erschien es nicht lohnend, die Abhängigkeit der genannten Größen mathematisch zu fassen. Zur Gewinnung eines Überblicks wurde die Regressionskurve entsprechend den $15 \mathrm{~mm}$ Längengruppen in Teilabschnitte zerlegt und für jeden dieser Teilabschnitte unter Annahme von Linearität die Regressionsgerade berechnet und in der graphischen Darstellung eingetragen. Die Werte Kopflänge/Otolithenlänge wurden unter Benutzung der Kurve Totallänge/Kopflänge in Anlehnung an die 15-mm-Gruppen eingeteilt. Tab. 2 gibt eine Zusammenfassung der Regressionsberechnungen. Die Signifikanz der Unterschiede im Anstieg aufeinanderfolgender Teilgeraden wurde bestimmt. Wo in der Tabelle die Angabe des P-Wertes fehlt, hatte sich bei der Varianzanalyse ergeben, daß die Varianz innerhalb der beiden betrachteten Längengruppen (Binnenvarianz) 
größer oder annähernd gleich groß wie die Varianz zwischen den Gruppen war und damit die Unterschiede zwischen den Regressionskoeffizienten nicht signifikant waren. In solchen Fällen wurde die beiden Längengruppen gemeinsame Regression berechnet und in Tabelle und graphischer Darstellung (dort anstelle der Einzelgeraden) eingetragen.

Otolithenlänge : Totallänge (Abb. 4). Während der Larvalentwicklung wächst der Otolith relativ langsam, von Beginn der Metamorphose bis zu etwa $85 \mathrm{~mm}$ Totallänge nimmt die Otolithengröße stark zu. Bei den größeren Tieren streuen die Otolithenlängen sehr stark, so daß sich bisher bei ihnen keine verläßlichen Aussagen über die Beziehung von Otolithenlänge zu Totallänge machen lassen. Eine Verlangsamung des Otolithenwachstums bei den 85-100 $\mathrm{mm}$ langen Jungheringen, der später wieder eine Beschleunigung mit einem der Larvalentwicklung ähnlichen Regressionskoeffizienten folgt, ist für das vorliegende Material statistisch gesichert.

Kopflänge : Totallänge (Abb. 5). Die Beziehung zwischen Kopflänge und Totallänge ist ebenfalls nicht linear, ihr Kurvenverlauf ähnelt derjenigen von Otolithenlänge zu Totallänge. Das Kopfwachstum während der Metamorphose ist erheblich schneller als während des Larvenlebens, verlangsamt sich dann aber wieder. Bei Tieren über $85 \mathrm{~mm}$ zeigt das Kopfwachstum eine weitere Verzögerung. Für die Längengruppe von 100-130 mm liegt kein ausreichendes, einheitliches Material vor, immerhin scheint hier wie beim Otolithenwachstum eine erneute Beschleunigung eingetreten zu sein. $\mathrm{Zu}$ ähnlichen Ergebnissen kam HARDER (1952) an Jungheringen aus der Tade. Auffallenderweise fand sich auch bei Harders Messungen eine Unstetigkeit im Kurvenverlauf bei etwa $80-90 \mathrm{~mm}$ Totallänge, über deren Ursache noch nichts bekannt ist.

Bezüglich des Larven- und Metamorphosestadiums stimmen unsere Beobachtungen mit den Feststellungen von Hemcke (1897) überein, der schreibt: „Bezeichnend für das Larvenstadium des Herings ist also die außerordentliche relative Länge des gleichzeitig sehr niedrigen Rumpfes oder Mittelkörpers und die Kürze des Kopfes und Schwanzes. Das Ubergangsstadium ist charakterisiert durch das starke Längenwachstum von Kopf und Schwanz und die relative Verkürzung des Rumpfes." Anschließend während des "ersten Jugendstadiums" (HerNcke) scheint der Kopf beim Nordseehering jedoch langsamer zu wachsen als HernckE für den Kieler Hering angibt.

Otolithenlänge : Kopflänge (Abb. 6). Die Kurve in Abb. 6 zeigt im Gegensatz zu den bisher betrachteten nur einen Knick. Bis etwa $13 \mathrm{~mm}$ Kopflänge ist das Otolithenwachstum langsamer als bei den höheren Kopflängenmaßen. Während sich bei den Beziehungen Otolithenlänge : Totallänge und Kopflänge : Totallänge fast sämtliche 15-mm-Gruppen in ihren Regressionskoeffizienten signifikant unterscheiden, gilt für die Beziehung Otolithenlänge zu Kopflänge weitgehend Isometrie. Nur die Gruppen 7,0-13,0 mm Kopflänge sind gegenüber $13,1-16,3 \mathrm{~mm}$ signifikant verschieden. Die Grenze zwischen diesen beiden Gruppen fällt mit etwa $55 \mathrm{~mm}$ Totallänge, d.h. mit dem Ende der Metamorphose zusammen.

\section{Die Größe des Zentralfeldes}

Wie beim Gesamt-Otolithen bestimmten wir auch beim Zentralfeld den größten Durchmesser. Postuma \& Zijlstra gaben bei der Bearbeitung der Otolithen von Nordseeheringen des zweiten Lebensjahres den Radius des 


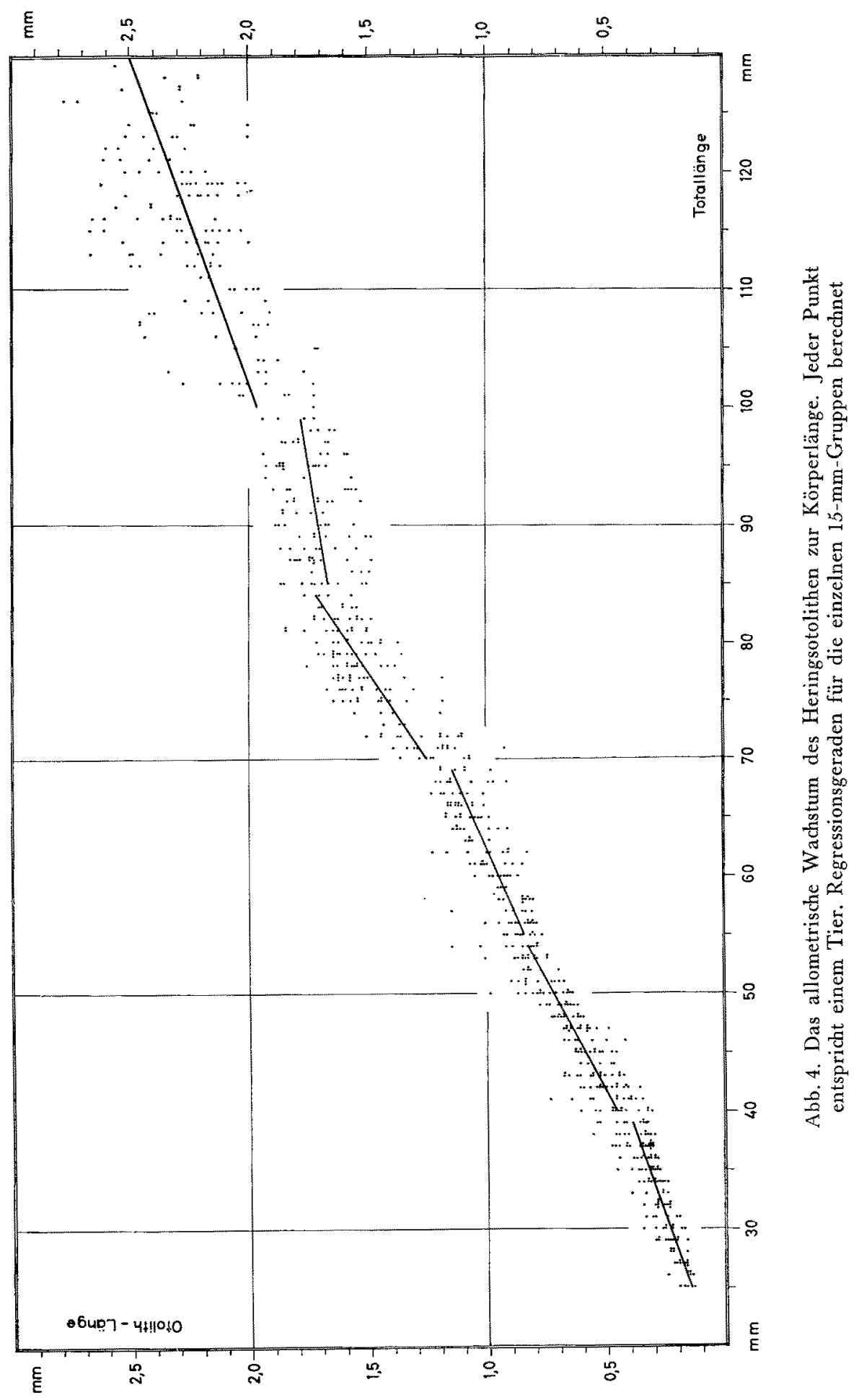




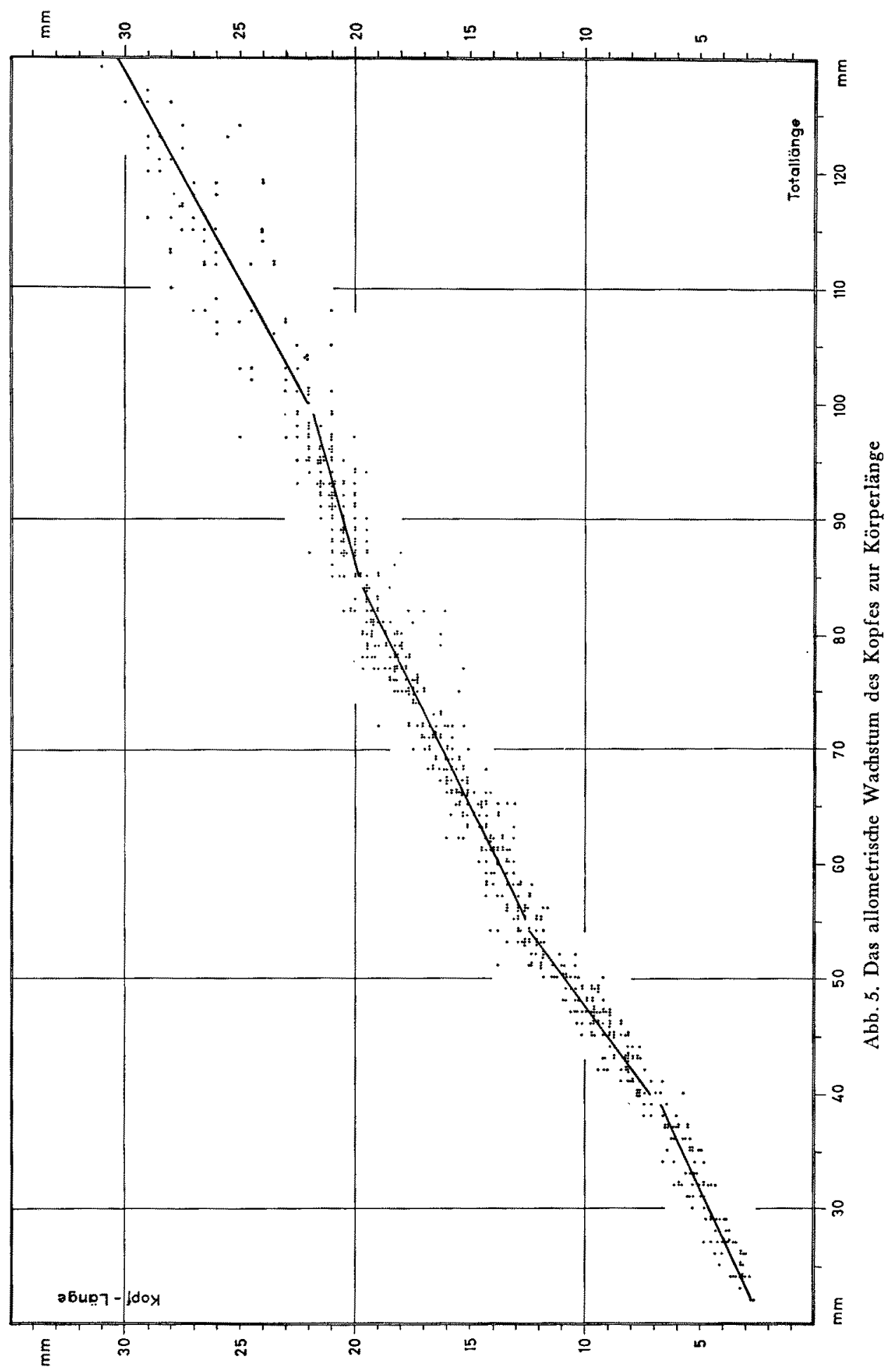




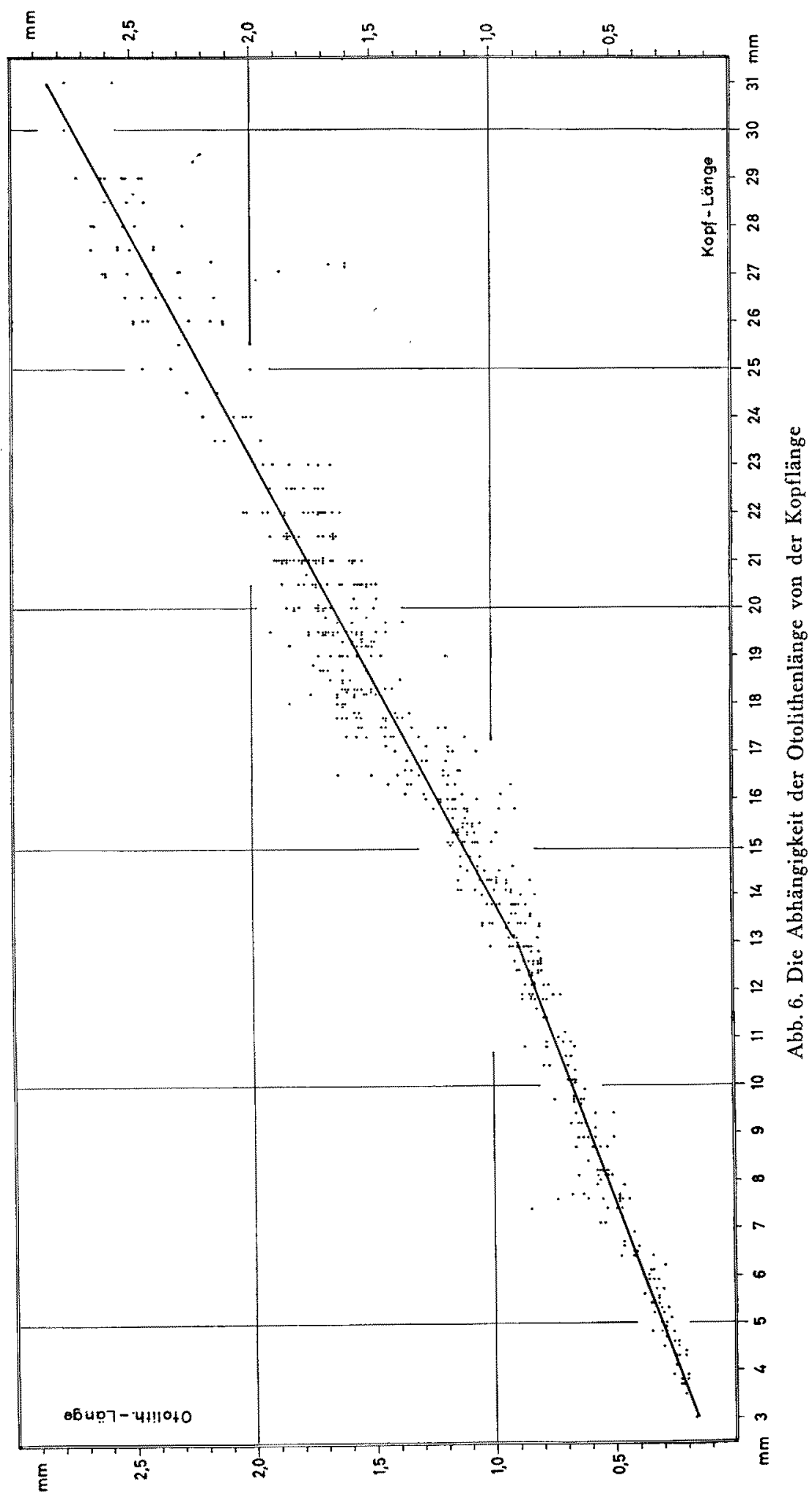


Zentralfeldes an. Infolge der unregelmäßigen Form des Zentralfeldes ist es nicht zu erwarten, daß der größte Radius nur die Hälfte des Durchmessers ausmacht. Immerhin ist auffallend, daß die von Postuma \& Zijustra angegebenen Radiuswerte nicht deutlich geringer als unsere Durchmesserdaten sind. Diese Diskrepanz dürfte - zumindest teilweise - auf das Auftreten einer Ubergangszone zwischen Zentralfeld und Sommerwachstum zurückzuführen sein (Abb. 7a). Bei 82 Otolithen war die Ubergangszone so scharf ausgebildet,

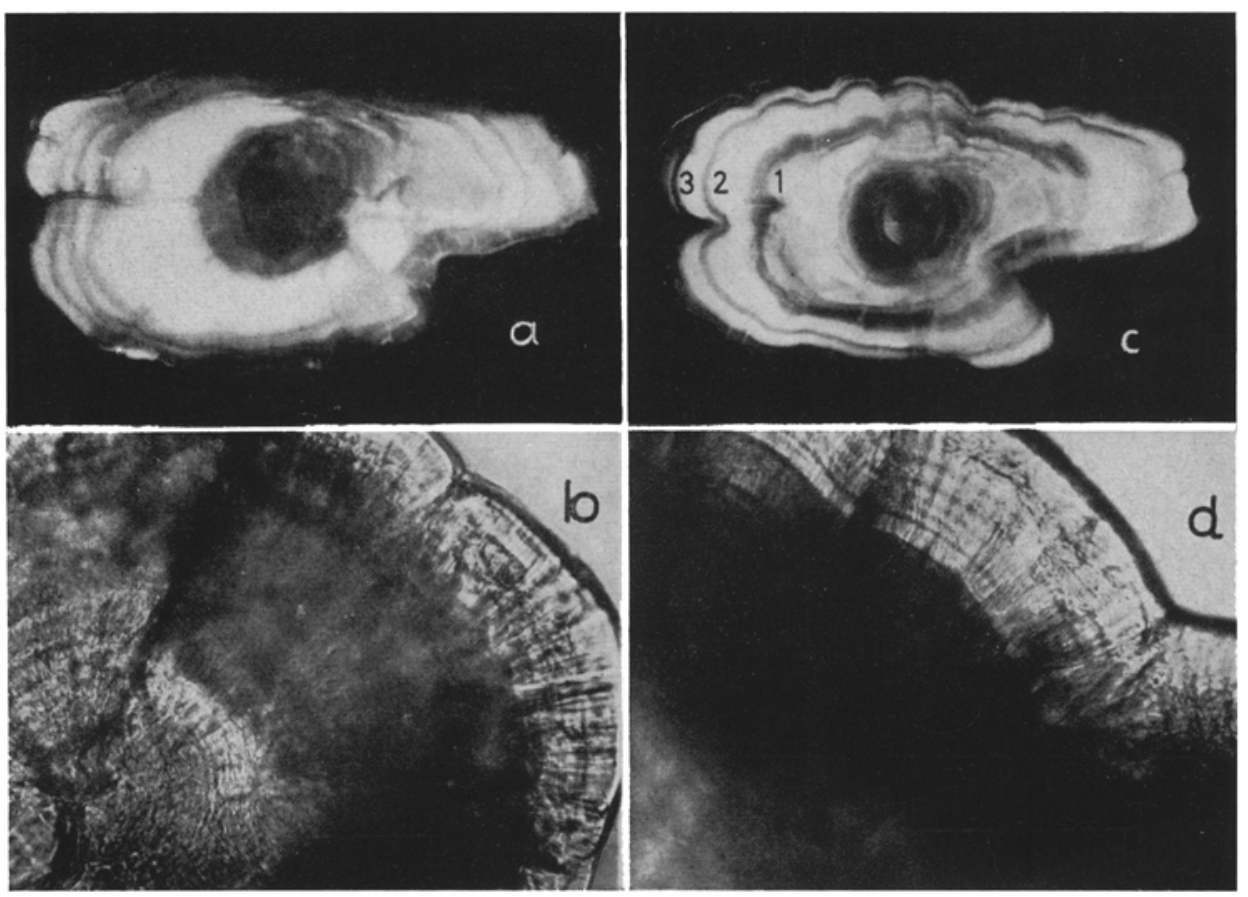

Abb. 7. a) Otolith $(\varnothing=1,64 \mathrm{~mm})$ mit Übergangszone am Rande des Zentralfeldes. Junghering, O-Gruppe (?), $78 \mathrm{~mm}$, Probe IX, 21. 7. 57. Auflicht. - b) Ausschnittsvergrößerung eines Otolithen mit opakem Ring und hyalinem Rand. Junghering, O-Gruppe, $67 \mathrm{~mm}$, Probe XII, 27. 6. 57. Durchlicht. - c) Otolith $(\varnothing=2,41 \mathrm{~mm})$ eines $108 \mathrm{~mm}$ langen Jungherings aus der Elbmündung. Alter ungewiß, Probe XXII, 14. 8. 58. Auflicht. Nummern der hyalinen (hier dunklen) Ringe s. Text. - d) Ausschnittvergrößerung des Otolithen eines Jungherings. O-Gruppe, $90 \mathrm{~mm}$, Probe XII, 27.6.57. Durchlicht

daß3 das Zentralfeld sowohl mit als ohne Übergangszone vermessen werden konnte. In andern Fällen waren die Grenzen zwischen Zentralfeld, Übergangszone und Sommerring nicht klar zu erkennen, ja es war am photographischen Bild nicht zu entscheiden, ob es sich um ein sehr großes Zentralfeld oder um ein Zentralfeld mit Ubergangszone handelt. Als Grenze des Zentralfeldes wurde - soweit vorhanden - der innere Rand der Übergangszone, in allen anderen Fällen der innere Rand des Sommerrings angegeben. Bei Otolithen älterer Heringe mit stark ausgebildetem Sulcus war es mitunter nicht zu entscheiden, ob die Aufhellung auf der Sohle des Sulcus cranial vom Zentrum durch die geringe Dicke des Otolithen an dieser Stelle oder durch eine hyaline Zentralfeldstruktur hervorgerufen wurde. Die von Postuma \& ZiJLSTRA beschriebene radiäre Struktur des Zentralfeldes bei Heringen der 
I-Gruppe, die mit Beginn des opaken Ringes in eine unregelmäßige Netzstruktur übergeht, konnten wir auch unter stärkerer Vergrößerung nur bei wenigen der O-Gr.-Otolithen erkennen. Häufiger fanden wir, daß das Zentralfeld eine körnige, konzentrisch angeordnete Struktur aufweist, wie sie schon an den Otolithen von Heringslarven zu sehen ist (Abb. 8a). Die bei schwacher Vergrößerung erkennbaren hyalinen Zonen ließen sich auch bei stärkerer Vergrößerung nicht eindeutig auf Strukturunterschiede zurückführen, allerdings ist in hyalinen Zonen (abgesehen vom Zentralfeld) die radiäre Anordnung langgestreckter Teilchen oft deutlicher zu erkennen als in den opaken Ringen (Abb. 7b). Dies stimmt mit Immermanns (1908) Auffassung von der Anord-



Abb. 8. a) Otolith $(\varnothing=0.13 \mathrm{~mm})$ einer $25 \mathrm{~mm}$ langen Heringslarve. Probe V, 3. 4. 57. Durchlicht. - b) Otolith $(\varnothing=1,44 \mathrm{~mm}$ ) eines $72 \mathrm{~mm}$ langen Jungherings, O-Gruppe, Probe XIX, 10.-12. 6. 58. Durchlicht

nung der Kalkelemente im Otolithen gut überein. Die hyalinen Zonen werden von gleichmäßig radiär verlaufenden Kalknadeln gebildet, während diese in den opaken Zonen durch eine Störung im Antagonismus der organischen und anorganischen Bauelemente des Otolithen nicht streng radiär gelagert werden. Auch das Polarisationsmikroskop lieferte keine Hilfe bei der Abgrenzung des Zentralfeldes. Durch Einschaltung von Polarisator und Analysator wurden lediglich die feinen konzentrischen Ringe auf der Oberfläche und der dünne Rand des Otolithen deutlicher. An die Exaktheit unserer Angaben über die Zentralfeldgröße können keine großen Ansprüche gestellt werden, Wiederholungsmessungen ergaben mitunter erhebliche Abweichungen in den Meßdaten.

Die Untersuchungen über die Größe des Zentralfeldes waren vorerst auf die Beantwortung folgender Fragen gerichtet: 1. Andert sich die Größe des Zentralfeldes während des späteren Wachstums des Otolithen, etwa durch das Einlagern oder Uberlagern opaker Substanz? 2. In welchem Monat des Jahres ist die Bildung des Zentralfeldes bei den Doggerbankheringen abgeschlossen?

Die Kurve der Frequenzen der Zentralfeldlängen ist deutlich bimodal mit einem Hauptmaximum etwa bei $0,22 \mathrm{~mm}$ und einem zweiten Maximum etwa bei $0,43 \mathrm{~mm}$ (Abb. 9). In dieser Kurve sind nicht diejenigen Otolithen enthalten, bei denen sowohl das Zentralfeld allein als auch Zentralfeld + Uber- 
gangszone gemessen werden konnten. Für diese Otolithen ergab sich $0,24 \mathrm{~mm}$ als mittlerer Durchmesser des Zentralfeldes ohne Übergangszone, während der Mittelwert für Zentralfeld + Übergangszone $0,47 \mathrm{~mm}$ betrug. Demnach können wir annehmen, daß wir es nur im linken Kurvenabschnitt der Abb. 9 mit typischen Zentralfeldern zu tun haben, während die hohen Werte Zentralfeld und Übergangszone umfassen, indem bei diesen Tieren eine Übergangszone vorlag, die nur nicht genügend deutlich vom Zentralfeld geschieden war. Die bimodale Verteilung aus Abb. 9 macht sich auch bei der Auftragung der Beziehung Zentralfeldgröße/Totallänge bemerkbar (Abb. 10).

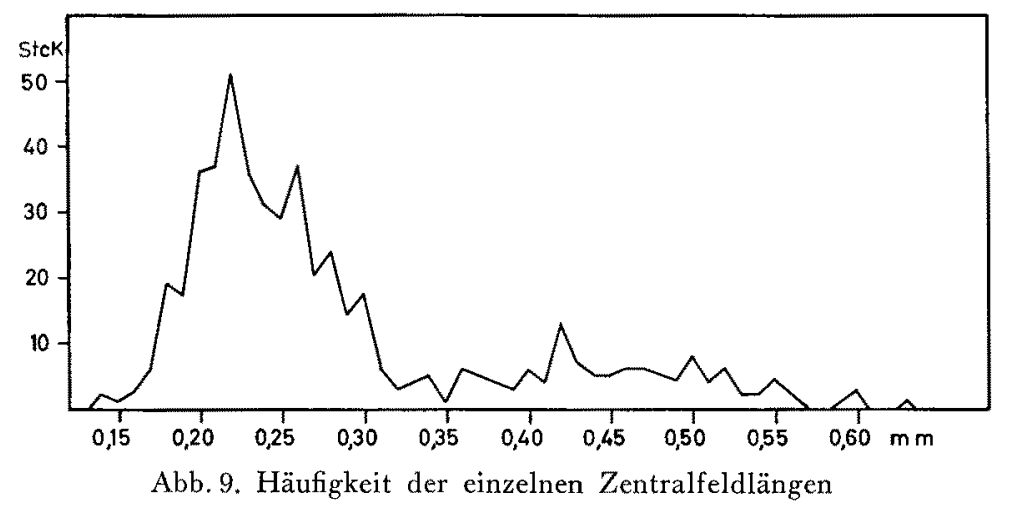

Nach Abb. 10 bleibt die Größe des Zentralfeldes und der Übergangszone während der Jugend des Herings konstant. Lediglich bei den kleinsten Otolithen traten in vermehrter Anzahl auffallend kleine Zentralfelder auf. Möglicherweise ist bei diesen Otolithen die Zentralfeldbildung noch nicht abgeschlossen. Der feine Rand, der sich bei ihnen um das Zentralfeld legt, wäre dann nicht als Ansatz der opaken Zone zu denken. Eine hier nicht wiedergegebene Erweiterung der Darstellung bis auf mehr als $200 \mathrm{~mm}$ Totallänge zeigt, daß sich die Zentralfeldgröße auch nach dem Ende des ersten Lebensjahres nicht mehr verändert. Auf Grund dieser Feststellung läßt sich indirekt der Abschlußtermin des Zentralfeldwachstums bestimmen: Nach Abb. 9 ist das Zentralfeld bei Doggerbankheringen etwa $0,20-0,26 \mathrm{~mm}$ im Durchmesser lang. Otolithen dieser Größe finden wir in Heringslarven von 28-30 mm Totallänge. Nach den Untersuchungen von BücKMann \& Hempel erreicht das Gros der Doggerbanklarven diese Länge normalerweise im März. Zentralfeld + Übergangszone sind etwa $0,45 \mathrm{~mm}$ groß, was einer Totallänge des Herings von $40 \mathrm{~mm}$ entspricht. Doggerbanklarven dieser Größe wurden Ende April in der Deutschen Bucht und ihren Küstengewässern gefangen. - Die als mutmaßliche Downslarven in der Abb. 10 hervorgehobenen kleinen Heringslarven unterscheiden sich in der Zentralfeldgröße nicht von den Doggerbanklarven. Das Material ist jedoch zu klein und die Herkunft der Larven nicht sicher genug, um aus dieser Feststellung Schlüsse zu ziehen, die denen von Postuma \& Zijlstra widersprechen. Auch bei den älteren Heringen, die möglicherweise bezüglich ihrer Herkunft inhomogen waren, indem sie teils aus der westlichen Nordsee, teils aus dem englischen Kanal stammen können, fand sich keine zweigipflige Verteilung der Zentralfeldgröße. Aber auch hier wollen wir uns einstweilen aller Schlußfolgerungen enthalten. 


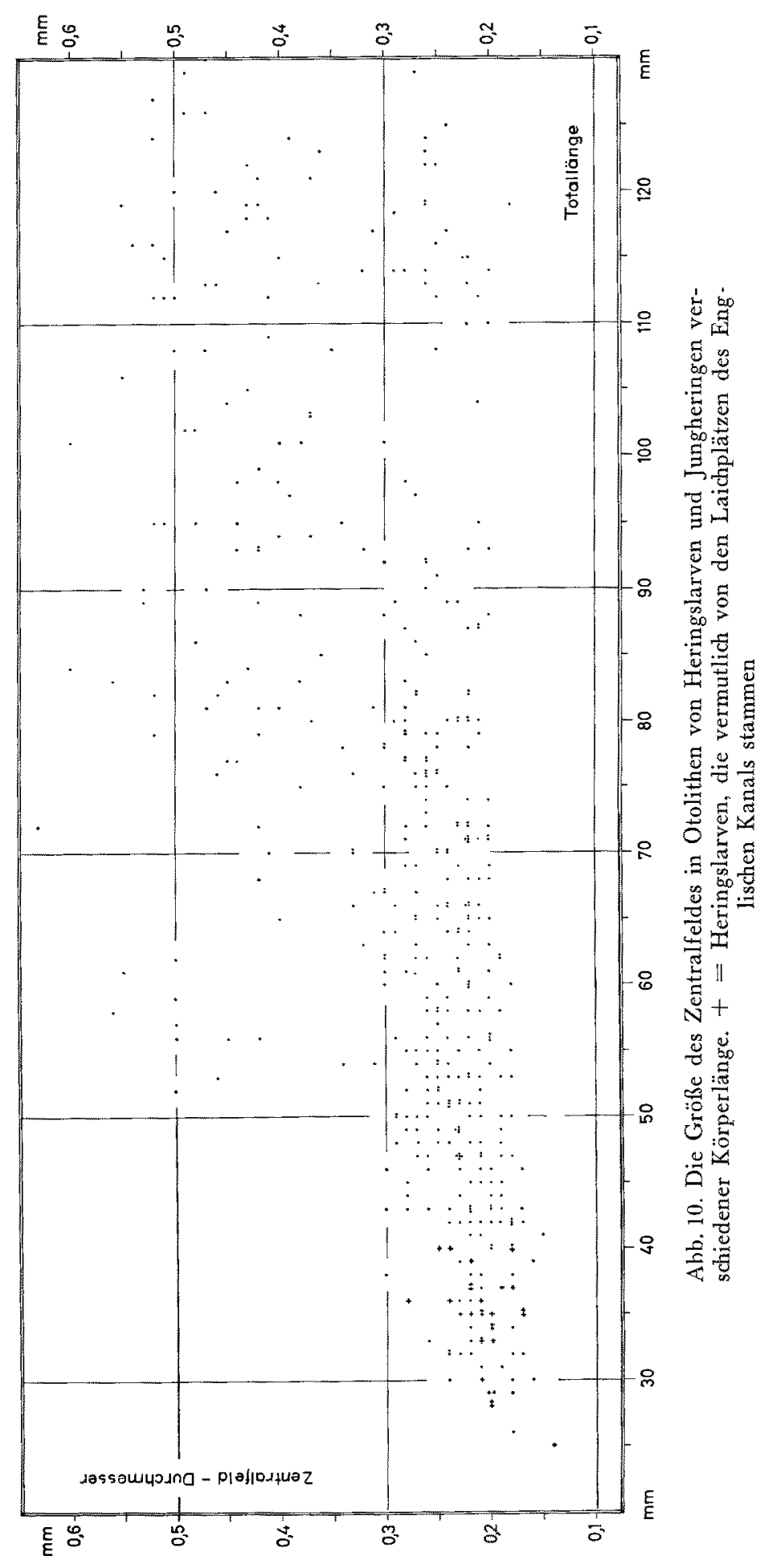


$\mathrm{Zu}$ ähnlichen Zeitangaben über das Ende der Zentralfeldentwicklung kommt man bei der Betrachtung der Otolithen in Heringslarven. In einzelnen Fällen muß es schon sehr früh im Jahr zur Bildung einer opaken Zone kommen, denn bei 4 von insgesamt $30 \mathrm{im}$ Februar/März gefangenen Larven wurden von einem opaken Rand umschlossene Zentralfelder gefunden. Zwei von diesen Larven waren sehr groß (42 und $46 \mathrm{~mm}$ ), ihre Zentralfelder aber auffallend klein $(0,18$ bzw. $0,17 \mathrm{~mm})$. Das Zentralfeldwadsstum muß bei ihnen schon im Winter zu Ende gegangen sein. Nach ihrer Körperlänge zu urteilen, stammen diese Larven wahrscheinlich von den Laichplätzen der nördlichen Nordsee. Leider liegt bisher kein größeres Material aus dem März vor. Bei den Anfang April (Probe V) gefangenen Larven war die äußere Begrenzung des Zentralfeldes bereits in vielen Fällen zu erkennen. In der Probe V waren die Otolithen meist nur wenig größer als ihre Zentralfelder, die Bildung einer opaken Zone deutete sich gerade am Otolithenrand an, während bei den Otolithen aus Probe VII (24. 4. 58) die opake Zone bereits einen breiten, kräftigen Ring bildete. Dennoch können wir den Abschluß der Ausbildung des Zentralfeldes bei den hier untersuchten Heringslarven auf Ende März ansetzen. In Probe V waren die kleinsten Larven mit abgeschlossenem Zentralfeld $28 \mathrm{~mm}$ lang. Bei einer Zentralfeldgröße von $0,20 \mathrm{~mm}$ maßen ihre Otolithen $0,22 \mathrm{~mm}$ im Durchmesser, der kleinste Otolith $(0,18 \mathrm{~mm})$ hatte ein Zentralfeld von $0,16 \mathrm{~mm}$. Die ersten Tiere, bei denen auch die Ubergangszone vermessen werden konnte, fanden sich in Probe VIII vom 9. 5. 1958. Es muß noch erwähnt werden, daß nur bei einem Teil der Otolithen von Heringslarven, die im April und Mai gefangen wurden, die Bildung des opaken Ringes zu erkennen war. Im durchfallenden Licht heben sich in den dünnen Larvenotolithen die opaken Ringe oft nicht genügend vom Zentralfeld ab. Dann wird die Bildung opaker Zonen erst bei größeren Otolithen sichtbar, und zwar zuerst im Ansatz des Rostrum und halbkreisförmig am caudalen Rand des Zentralfeldes (Abb. 8b).

\section{Ringbildungen im Otolithen während des ersten Lebensjahres}

ImmermanN hat darauf hingewiesen, daß der Sommerring aus einer größeren Anzahl opaker Zonen zusammengesetzt ist, zwischen denen durchsichtige Zonen liegen. Die Otolithen der jungen Heringe zeigen diese Erscheinung sehr deutlich. In den Monaten April und Mai ist die Ausbildung opaker Strukturen am stärksten, wie das auch Immermann für die Scholle beschreibt. Während der folgenden Sommermonate werden einzelne schmalere, opake Zonen zwischen stärker hyalinen Strukturen angelegt. Wie scharf mitunter die Bildung der ersten opaken Zone zu Beginn des Sommers abgebrochen wird, zeigt der Otolith in Abb. $7 \mathrm{~d}$, der aus einem im Juni gefangenen Tier stammt. Schon Lissner hat die scharfe äußere Begrenzung der ersten opaken Zone bei großen Larven beobachtet. Oftmals finden sich zwischen den opaken so klare hyaline Zonen, daß man bei Unkenntnis der Fischgröße geneigt wäre, auf ein mehrjähriges Alter der Jungheringe zu schließen (Abb. 7a). In späteren Lebensjahren ist die Aufsplitterung des opaken Ringes erheblich geringer als während des ersten Sommers. Bei manchen Otolithen scheint nach schwacher Ringbildung während des Sommers im Spätsommer 
oder Herbst noch einmal eine kräftige opake Zone angelegt zu werden, auf die unmittelbar ein scharf ausgeprägter erster hyaliner Winterring folgt. Bei anderen Otolithen ist es nicht leicht, sich für diese Deutung zu entscheiden, statt die auf das Wachstum des 1. Frühjahrs folgende breite, vorherrschend hyaline Struktur bereits als 1 . Winterring anzusprechen. So war es bei Jungheringen, die im August 1958 in der Jade und in der Elbmündung gefangen worden waren, nicht möglich, anhand des Otolithenbildes sicher zu entscheiden, ob die 9-13 cm langen Tiere am Ende des ersten, zweiten oder gar des dritten Lebensjahres standen. Abb. 7c zeigt den Otolithen eines Herings aus der Elbmündung (Probe XXII vom 14. 8. 58), der auf Grund seiner Körpergröße $(108 \mathrm{~mm})$ als einjährig, d. h. im Herbst 1957 geschlüpft, angesehen werden könnte. Dann wäre die hyaline Zone 1 am Ende des Frühjahrswachstums 1958, die Zonen 2 und 3 im Sommer 1958 angelegt worden. Es liegt andererseits nahe, zumindest die Zone 2 als Winterring zu werten und damit den Junghering dem Jahrgang 1956 zuzuweisen. Sieht man die Zonen 1 und 2 als echte Winterringe an, so rechnet man das Tier dem Jahrgang $1955 \mathrm{zu}$.

Unter den 47 daraufhin untersuchten Jungheringsotolithen der Probe XXII zeigten 16 Otolithen in sehr klarer Form den weiten hyalinen Ring nach schmaler opaker Zone, während 9 Otolithen durch ein breites Sommerwachstum ausgezeichnet waren, auf das ein schmaler hyaliner Ring folgte. Der Abstand von der äußeren Begrenzung des hyalinen Ringes zum Otolithenrand war bei den Otolithen mit weitem hyalinen Ring bedeutend größer als bei dem anderen Typ. In den meisten Fällen war die Bildung opaker Strukturen am stärksten in der Umgebung des Zentralfeldes und nahe dem Otolithenrand. Zwischen beiden Typen gab es auch Übergänge, indem auf das erste Frühjahrswachstum eine semi-opake Zone folgte, die zum Rande hin durch einen \pm scharfen hyalinen Ring gegen eine stark opake Zone abgegrenzt wurde. Die Ahnlichkeit der hier beschriebenen weiten und schmalen hyalinen Zonen in Verbindung mit schmalen bzw. breitem Sommerring mit den von Parrish \& Sharman beschriebenen Otolithentypen der ", wide first winter zone " und "thin first winter zone" ist auffallend. Deutliche Unterschiede in der Körpergröße bestanden zwischen den Heringen der beiden verschiedenen Otolithentypen in Probe XXI und XXII nicht. Es wäre denkbar, daß die Heringe dieser beiden Proben älter sind, als wir auf Grund ihrer Körperlänge annehmen. Es handelt sich bei ihnen um Tiere, die unter den abnormen Lebensbedingungen der Ästuare aufwuchsen. Es sei hervorgehoben, daß bei Proben aus der Deutschen Bucht die Schwierigkeiten in der Altersbestimmung von Jungheringen nur in beschränktem Maße auftraten. Es bedarf noch weiterer Untersuchungen, um die verschiedenen Ringbildungen im Heringsotolithen auf die Umwelteinflüsse zurückzuführen, die sich im Wechsel der Jahreszeiten und der Fischwanderungen ändern.

\section{Zusammenf as sung}

Etwa 1000 Heringslarven und Jungheringe aus den Fängen der Jahre 1957 und 1958 wurden untersucht. Die Proben bestanden in erster Linie aus den Abkömmlingen der im Sommer/Herbst laichenden Bankheringe der mittleren und nördlichen Nordsee. Downslarven und -jungheringe spielten daneben nur eine geringe Rolle. Die Ausmessung des Otolithen und seines Zentralfeldes erfolgte auf Grund von Photographien. 
Die Ausformung des Otolithen wird anhand von Umrißzeichnungen beschrieben (Abb. 3).

In den Abb. 4-6 sind die Allometrieverhältnisse Otolithenlänge : Totallänge, Kopflänge : Totallänge und Otolithenlänge : Kopflänge dargestellt. Die Allometrieverhältnisse von Kopf und Otolith zur Körperlänge ändern sich während des ersten Lebensjahres erheblich. Otolith und Kopf wachsen dagegen nach der Metamorphose annähernd isometrisch zueinander.

Die Ausmessung des Zentralfeldes stößt infolge seiner oft unscharfen Begrenzung auf erhebliche Schwierigkeiten. Bei vielen Otolithen liegt zwischen dem eigentlichen Zentralfeld und der ersten opaken Zone eine semi-opake Ubergangszone. Die Strukturen der Otolithen von Heringslarven werden kurz beschrieben.

Nach Abschluß der Zentralfeldbildung scheint sich die Größe des Zentralfeldes - zumindest während der ersten beiden Lebensjahre - nicht zu verändern (Abb. 10). Längen-Rückenberechnungen an Zentralfeldern und direkte Beobachtungen ergaben, daß die Larven des Bankherings Ende März in der Deutschen Bucht mit der Bildung des ersten opaken Sommerringes beginnen.

Der erste opake Ring des Heringsotolithen besteht wie bei anderen Fischen aus einer Aufeinanderfolge von Zonen unterschiedlicher Transparenz. Im Frühjahr werden sehr kräftige opake Strukturen angelegt, denen später transparentere folgen. Für manche Otolithen - besonders bei Jungheringen, die im August im Küstenwasser gefangen worden waren - war es nicht zu entscheiden, ob die diffuse hyaline Zone, die bereits im Sommer angelegt wird, als Sekundärring oder als erster "Winterring" bewertet werden muß.

Weitere Untersuchungen über die Struktur der Otolithen von Heringen verschiedener Herkunft und über das Wachstum der Jungheringe der Deutschen Bucht sind in Vorbereitung.

\section{Summary}

This preliminary communication deals with the development of the herring otolith mainly during the first year of life. The material was collected in 1957 and 1958 within the German Bight. It mainly consists of the offspring of summer/autumn spawning Bank herring.

The different developmental stages of otoliths of $\mathrm{O}$-group herrings are shown in fig. 3 . The typical outline of the herring otolith is reached at about $90 \mathrm{mms}$ of total length. The otolith length (largest diameter) was determined from photographs. The allometry of the otolith growth to body size shows four main different phases: 1 . The larval stage up to metamorphosis, 2. the growth during and immediately after metamorphosis, 3. a disturbance at about $80-90 \mathrm{mms}$ of total length and 4 . after that a growth similar to that of the larval stage (fig. 4). These conditions are about the same as in the relation of head to body length (fig. 5). Therefore the relation between head and otolith remains constant in the main. Only one significant break of the curve was found: $U_{p}$ to metamorphosis the otolith grows negatively allometric. Afterwards isometry is nearly established (fig. 6). The measurements on size of the otolith centre were hampered by the lack of a sharp contour of the centre in many otoliths. A transition zone lying between the centre and the opaque ring and the structure of larval otoliths is described (fig. $7-8$ ).

There is no difference between the size of otolith centres just after they have been formed and about 1 year later (fig. 10). Back calculations and direct observations showed, that the formation of the first opaque ring starts in larvae of about $28-30 \mathrm{mms}$ at the end of March.

The first opaque zone of the herring otolith consists of a succession of opaque and hyaline rings. In some otoliths it is not possible to come to a decision wether the broad zone of semi-opaque or hyaline elements following the first strong opaque ring has not to be reckoned 
as secondary ring meaning a part of the summer zone followed by a further opaque zone in late summer or rather as first "winter ring".

Further investigations are planned on otoliths of different origin and on the growth pattern of young herring in the German Bight.

\section{Literaturverzeichnis}

Bückmann, A. \& G. Hempel, 1957: Untersuchungen an der Heringslarvenbevölkerung der Innenjade. Helgol. Wiss. Meeresunters. 6, 52-70.

Ein a rs o n, H., 1951: Racial analyses of Icelandic herrings by means of the otoliths. Cons. Intern. Explor. Mer, Rapp. et Proc.-Verb. 128, 55-74.

Harder, W., 1952: Zur postembryonalen Entwidklung des Darmes beim Hering (Clupea harengus L.). Z. Anat. Entwicklungsgesch. 116, 379-398.

Heincke, F., 1897: Naturgeschichte des Herings I. Wiss. Abhandl. Seefischerei I, H. l.

I $m \operatorname{mermann}, F ., 1908$ : Beiträge zur Altersbestimmung der Fische II. Wiss. Meeresunters., Abt. Helgoland, N.F. 8, 131-175.

Jenkins, J. T., 1902: Altersbestimmung durch Otolithen bei den Clupeiden. Wiss. Meeresunters., Abt. Kiel, N.F. 6, 81-122.

Lissner, H., 1925: Die Altersbestimmung beim Hering mit Hilfe der Otolithen. Ber. Dtsch. Wiss. Komm. Mceresforsch. 1, 181-198.

Parrish, B. B. \& D. P. Sharman, 1958: Some remarks on methods used in herring "racial" investigations with special reference to otolith studies. Cons. Intern. Explor. Mer, Rapp. et Proc.-Verb. 143, II, 66-80.

Postuma, K. H. \& J. J. Zijlstra, 1958: On the distinction of herring races in the autumn- and winter-spawning herring at the southern North Sea and English Channel by means of the otoliths and application of this method in tracing the offspring of the races along the continental coast of the North Sea. Cons. Intern. Explor. Mer, Rapp. et Proc.-Verb. 143, II, 130-133. 